\title{
Analítica del aprendizaje y las neurociencias educativas: nuevos retos en la integración tecnológica
}

\author{
Arturo Corona Ferreira 1 (이 @ \\ Mijael Altamirano ${ }^{2}$ (D) @ \\ María de los Ángeles López Ortega ${ }^{1}$ (D) @ \\ Oscar Alberto González González ${ }^{1}$ (D) @ \\ ${ }^{1}$ Universidad Virtual del Estado deMichoacán (UNIVIM), México; ${ }^{2}$ Instituto Politécnico Nacional (IPN), Mexico.
}

Resumen. La analítica del aprendizaje (LA) tiene como objetivo comprender y optimizar el aprendizaje y los entornos en los que se produce. Dada la compleja naturaleza de los procesos de aprendizaje, se ha hecho necesario usar herramientas de diversos campos de investigación que permitan obtener, describir, analizar e interpretar datos sobre los alumnos, durante sus procesos de aprendizaje y en sus contextos. Más que tomar métodos o técnicas aisladas para el estudio de este proceso, la analítica del aprendizaje está comenzando a integrar las perspectivas de otros campos para lograr una investigación del aprendizaje con una visión transdisciplinaria. Uno de estos campos es el de la neurociencia, específicamente la relacionada con el aprendizaje o neurociencia educativa. El objetivo de esta investigación es explorar las implicaciones y retos del uso de las tecnologías por medio de los electroencefalogramas (EEG) -tradicionalmente empleados en estudios neurológicos- en conjunto con las analíticas del aprendizaje para el estudio de los procesos de aprendizaje en los estudiantes.

Palabras clave: analíticas del aprendizaje; neurociencias; retos educativos.

Analítica da aprendizagem e a neurociência educacional: novos desafios na integração tecnológica

Resumo. A Analítica da Aprendizagem (LA) tem como objetivo compreender e otimizar a aprendizagem e os ambientes nos quais ela ocorre. Dada a complexa natureza do processo de aprendizagem, tornou-se necessário o uso de ferramentas de diversos campos de pesquisa para a obtenção, descrição, análise e interpretação de dados sobre os alunos durante seus processos de aprendizagem e em seus contextos. Ao invés de usar métodos ou técnicas isoladas para o estudo deste processo, a Analítica da Aprendizagem está começando a integrar as perspectivas de outros campos para alcançar uma pesquisa de aprendizagem com uma visão transdisciplinar. Um destes campos é o da neurociência, especificamente aquela relativa à aprendizagem ou neurociência educacional. O objetivo desta pesquisa é explorar as implicações e os desafios do uso das tecnologias por meio do eletroencefalograma (EEG), tradicionalmente aplicado em estudos neurológicos, em conjunto com as Analíticas de Aprendizagem para o estudo dos processos de aprendizagem nos estudantes.

Palavras-chave: Analítica de aprendizagem; neurociência; desafios educacionais.

Analytics of Learning and Educational Neurosciences: challenges in technological integration

Abstract. The aim of learning analytics $(L A)$ is to understand and optimize learning and the environments in which it occurs. Given the complex nature of learning, it has become necessary to use tools from various fields of research to obtain, describe, analyze and interpret data about students, their learning processes and contexts. Rather than taking isolated methods or techniques for the study of this process, learning analytics is beginning to integrate the perspectives of other fields to achieve a research of learning with a transdisciplinary vision. One of these fields is neurosciences, specifically those related to learning or educational neurosciences. The objective of this research is to explore the implications and challenges of the use of EEG technologies (traditionally used in neurological studies) in conjunction with learning analytics for the study of students learning processes and their context.

Keywords: learning analytics; neuroscience; educational challenges. 


\section{Introducción}

El concepto de analítica del aprendizaje o LA (por sus siglas del inglés, Learning Analytics) es relativamente nuevo y, a grandes rasgos, se refiere al conjunto de procesos asociados con el análisis de datos relativos al aprendizaje.

Para comprender los conceptos propios de LA, y utilizarlos para la formulación de propuestas para el mejoramiento docente y aprendizaje, es necesario identificar y comprender de donde surge y como se contrasta con otras áreas de conocimiento como la ciencia de datos y las ciencias educativas (Reimann, 2016).

Según la Society for Learning Analytics Research (SoLAR), la analítica del aprendizaje se define concretamente como "la medición, recopilación, análisis e informe de datos sobre los alumnos y sus contextos, con el fin de comprender y optimizar el aprendizaje y los entornos en los que se produce" (SoLAR, s.f.).

De acuerdo con esta definición, la analítica del aprendizaje puede hacer uso de cualquier herramienta o campo que le permita obtener, describir, analizar e interpretar datos sobre los alumnos y sus procesos de aprendizaje; de esta manera, las analíticas del aprendizaje permiten crear conocimiento que contribuya a mejorar el proceso de aprendizaje y su entorno.

Otros autores definen las analíticas del aprendizaje en términos similares; entre ellos, vale la pena destacar a Siemens (2013), quien indica que las analíticas del aprendizaje involucran la recopilación, medida, análisis y reporte de datos sobre los estudiantes y sus contextos.

Adicionalmente, Stewart (2017) señala que, como campo de investigación, el concepto de LA se deriva de las actividades de las ciencias de datos (CD), cuyos campos de acción son: la minería de datos, la inteligencia artificial, la analítica web, así como la inteligencia comercial.

En este sentido, las analíticas del aprendizaje implican la integración de datos -de múltiples fuentes-, para informar la acción del uso de los sistemas de gestión educativa como los Learning Management System (LMS) (Stewart, 2017).

Por su parte, Baker y Siemens, Baker y Yacef, y Greller y Drachsler en Reimann (2016) presentan a LA como un campo de investigación joven impulsado, en especial, por la disponibilidad de datos relacionados con el 
aprendizaje en estudiantes; la disponibilidad de grandes conjuntos de datos ha generado interés en su análisis con el fin de mejorar la experiencia del aprendizaje.

Similarmente, Baker y Siemens en Banihashem et al. (2018) señalan que la creciente cantidad de datos educativos analizables es uno de los factores que condujeron a la aparición de LA. En efecto, recientemente se han publicado artículos con datos de decenas de miles de estudiantes; el tamaño y la disponibilidad de estos conjuntos de datos es el principal incentivo en favor del desarrollo de los estudios sobre LA.

En este sentido, Ferguson y Clow (2017) indicaron en la primera conferencia de Learning Analytics and Knowledge del año 2011, que los artículos recibidos incluían contenido, en los que predominaba la medición, la recopilación, el análisis e informes de datos sobre los estudiantes y sus contextos, y en las exposiciones de los resultados se identificaba que dichos trabajos buscaban demostrar como mediante analíticas lograban comprender y optimizar el aprendizaje.

En ese mismo evento, Banihashem et al. (2018) indican que se recibieron aportaciones de analíticas de datos sobre la medición, recopilación, análisis e informes de datos sobre los estudiantes y sus contextos. Contribuciones que fueron presentadas con el fin de comprender y optimizar el aprendizaje.

La recopilación de datos de los alumnos, cuando están en un contexto de aprendizaje, abren la puerta a nuevas interpretaciones acerca de lo que sucede durante la ejecución de los procesos de aprendizaje. Así lo demuestra Stewart (2017), al enfocarse en realizar LA, con el fin de emplear una técnica para modelar y generar perfiles de alumnos, así como la de abrir el medio para formular un aprendizaje personalizado y adaptable.

Banihashem et al. (2018) describen que, normalmente para los procesos de LA, los datos provienen de las interacciones con los sistemas, permitiendo arrojar datos durante el tiempo en que el alumno participa e, inclusive, cuando el alumno deja de usar contenidos. En dicha investigación los autores señalan, que el principal argumento de valor de LA es el tamaño de los datos, en referencia no solo a la cantidad de estudiantes analizados, sino a la cantidad y variedad de datos puntuales producidos por los estudiantes al interactuar con sistemas. 
Estos grandes conjuntos de datos, conocidos también como huellas digitales, se generan por los alumnos al hacer uso de mediaciones durante su aprendizaje. Esas huellas permiten formular las analíticas de los aprendizajes, proporcionando nuevas perspectivas para la mejora del aprendizaje y de la enseñanza a través de recursos tecnológicos (Tseng et al., 2014).

Banihashem et al. (2018), en base al National Research Council (2013), proponen que existen tres razones adicionales por las cuales el tamaño y la variedad de datos son la base de la verdadera analítica del aprendizaje. La primera de ellas es, que cuanto mayor sea el número de estudiantes, se podrá proponer una forma verdadera de generalización de hallazgos. La segunda razón, es referente a la variedad de datos, ya que cuanto más diverso sea el origen de los datos, aumenta exponencialmente el número de variables incluidas en el análisis y esto lleva a un mayor número de posibles relaciones entre ellas. Finalmente, en tercer lugar, los grandes conjuntos de datos dan pie a identificar eventos anómalos, ya que estos se alejan de los comportamientos regulares, los cuales se dan solo de forma esporádica o en pequeñas muestras de estudiantes.

Ahora bien, existe la ilusión de que la recopilación de grandes conjuntos de datos Ilevará finalmente a interpretaciones concluyentes sobre el aprendizaje, siendo en realidad las interpretaciones el desafío que el campo de LA debe abordar. Las grandes cantidades de datos son importantes, pero también requieren de atención los pequeños datos sobre individuos y tareas de aprendizaje particulares, ya que en los modelos de LA toda la información sobre los procesos de aprendizaje puede y debe ser considerada de manera integral (Lodge y Corrin 2017).

De acuerdo con Amo y Santiago (2017) en la analítica del aprendizaje se interpretan datos educativos mediante aproximaciones cuantitativas, sustentadas por los datos extraídos. Por lo tanto, la analítica del aprendizaje sirve para estudiar el pasado, el presente y el futuro del comportamiento de los alumnos.

Es necesario destacar que las analíticas del aprendizaje no tratan de cuantificar el aprendizaje, sino que tratan de entender el contexto en el que convive el alumno, con el propósito de descifrar, explicar y predecir el comportamiento de estos, ante la presencia de los contenidos, actividades y recursos, con el fin de identificar como mejorar todo el proceso de enseñanza y elevarlos en sus posibilidades (Amo y Santiago, 2017).

Todas estas definiciones contemplan la necesidad de estudiar, no solo los datos derivados de los procesos de aprendizaje en el sentido estricto, sino también los datos relacionados a los entornos o contextos de aprendizaje. 
A su vez, estas definiciones plantean de manera indirecta la necesidad de contar con herramientas provenientes de diversos campos de estudio para poder comprender los procesos de aprendizaje.

De acuerdo con Lodge y Corrin (2017) la analítica del aprendizaje tiene el potencial para cerrar algunas brechas en el campo de la educación, la psicología e incluso en la neurociencia aplicada a la educación, con el fin de tener un entendimiento más profundo del comportamiento de los estudiantes a medida que ellos aprenden en entornos educativos reales.

Sin embargo, para que los datos resultantes de la analítica del aprendizaje sean útiles y contribuyan a comprender los comportamientos del aprendizaje y los procesos de aprendizajes en los alumnos, es necesario determinar previamente los objetivos de la analítica del aprendizaje ¿qué se desea saber?, ¿qué es lo que ya se sabe? y ¿cómo se relaciona esto con el diseño del aprendizaje?

Solo cuando se determinan estos factores, es posible identificar qué datos se necesitan para lograr un correcto análisis de aprendizaje, con el fin de alcanzar los objetivos deseados, ya sea para diagnosticar desafíos en el aprendizaje, para identificar patrones de comportamiento o para descubrir procesos de aprendizaje (Lodge y Corrin, 2017).

En este sentido, Corrin et al. (2018) señalan que:

A medida que el campo de la analítica del aprendizaje madura, estamos empezando a ver un cambio en la forma en que los investigadores y las instituciones están hablando sobre la analítica del aprendizaje en el contexto del aprendizaje y la enseñanza. Desde sus raíces en el uso de datos de estudiantes para abordar la retención, el campo se está expandiendo y se está convirtiendo en verdaderamente transdisciplinario, recurriendo a metodologías y técnicas de análisis de múltiples disciplinas. (p.1)

Todo este proceso se encuentra enmarcado en un contexto educativo de rápida evolución y de cambios, profundamente influenciado por la tecnología. Es necesario aclarar que, para los fines de esta investigación, la tecnología impacta en el aprendizaje de dos maneras; en primer lugar, transformando los medios a través de los cuales se accede a la educación y modificando los contextos de aprendizaje. Por otro lado, hoy es posible emplear nuevos recursos tecnológicos, que nos permiten diseñar distintos acercamientos para hacer el seguimiento de los alumnos. 
Aún más, el auge de la tecnología, la simplificación de ésta y su implementación en estudios de analíticas del aprendizaje, permite no solo la integración de otros campos de investigación, sino desarrollar un verdadero enfoque transdisciplinario de las analíticas del aprendizaje, donde la recopilación, el análisis y la interpretación de datos, puede verse desde la perspectiva de otro campo de investigación sin dejar de lado los objetivos de las LA.

De acuerdo con Knight y Buckingham en Logde et al. (2018) el objetivo que persiguen las neurociencias y las analíticas del aprendizaje son las de tratar de comprender el aprendizaje a través de una lente específica basada en datos. En el campo de las neurociencias, la perspectiva de la analítica es biológica, mientras que dentro de la perspectiva de la analítica del aprendizaje es conductual.

A pesar de la diferencia entre ambas perspectivas, el problema subyacente en ambos campos es el mismo: la educación es un fenómeno complejo, multidimensional, contextual y social; por lo tanto, intentar comprender cómo se produce únicamente a través de una única perspectiva es insuficiente, e incluso problemático (Lodge et al., 2018).

\section{Analíticas del aprendizaje y las neurociencias educativas}

Durante la última década ha habido investigaciones importantes enfocadas a comprender y a entender el aprendizaje mediante estudios controlados, que están sentando las bases para una nueva ciencia del aprendizaje. En contraste, los grandes datos, a menudo generados en entornos tecnológicos, presentan a los investigadores datos más confusos de lo que es común. Sin embargo, LA ofrece una visión tentadora de los aspectos sociales, afectivos y metacognitivos del aprendizaje, como sucede en el trabajo auténtico y en los entornos escolares (Siemens, 2016).

Desde la investigación básica en ciencias cognitivas y neurociencia, incluida la investigación sobre el aprendizaje de animales, hasta los estudios experimentales y correlacionales en psicología educativa, y la investigación educativa sobre prácticas y pedagogías en el aula, se identifica al aprendizaje como una expresión que tiene lugar en múltiples niveles al mismo tiempo, los cuales se pueden identificar como eventos de aprendizaje producidos desde un estrato biológico con procesos neurofisiológicos, por medio de un estrato cognitivo que atiende al pensamiento racional y de conocimiento; y, un estrato sociocultural donde las herramientas junto con sus prácticas producen experiencias de aprendizaje (Reimann, 2016). 
Por su parte, Lodge et al. (2018) señalan que existen claros paralelos entre las analíticas del aprendizaje y la neurociencia educativa:

en ambos casos, se están produciendo grandes volúmenes de datos, los cuales son de utilidad para que los investigadores, estadísticos, maestros y otros que buscan inferir en el aprendizaje de estos conjuntos de datos. Por lo que es necesario la disponibilidad de abundantes datos, sobre cómo se produce el aprendizaje en el cerebro. Si bien, este esfuerzo ha brindado grandes conocimientos sobre el aprendizaje a nivel biológico, la extracción de significado de estos datos para la práctica educativa ha sido una tarea difícil" (p.14).

La disponibilidad de estos conjuntos de datos ha influido, según Logde et al. (2018), en el incremento de la investigación de estos temas, siendo el primer objetivo de los investigadores determinar cómo se produce el aprendizaje en el cerebro y cómo se correlaciona el aprendizaje a nivel cerebral y neuronal. Estas investigaciones han generado grandes avances desde la perspectiva biológica, mientras que desde el punto de vista educativo los avances han sido menores.

Uno de los grandes retos que presenta el estudio del aprendizaje, desde una perspectiva neurológica, es que no es posible inferir directamente de un estudio de imágenes cerebrales, lo que sucede en una persona en su totalidad; como tampoco es posible inferir datos puramente conductuales en un contexto sociocultural como el aula (Lodge et al., 2018).

Por si sola, la observación en el aula ya no es la herramienta definitiva para comprender los nuevos procesos de aprendizaje, esto es especialmente cierto en el caso de los entornos virtuales de aprendizaje, donde la observación es casi imposible.

Es así como LA se convierte en un recurso clave actual para analizar, visualizar, entender y mejorar los procesos de aprendizaje. La diferencia entre el seguimiento de un docente y las analíticas del aprendizaje es que estas últimas dotan de capacidad para observar y dar soporte a nivel global en una clase. En cambio, la capacidad observar del profesor solo le permite centrarse en un alumno a la vez (Amo y Santiago, 2017).

En este sentido, Ninaus et al. (2014) señalan que la analítica del aprendizaje es un campo muy prometedor para mejorar la educación, la enseñanza y el aprendizaje mediante la recopilación de datos de usuarios, especialmente aquellos datos de origen fisiológico. 
Pese a la relevancia que pudieran tener estos datos en el desarrollo de nuevos conocimientos en el área de las analíticas del aprendizaje, existen pocas investigaciones sobre el uso de rastros fisiológicos aplicados a las analíticas del aprendizaje.

De acuerdo con Zadina (2015) la neurociencia juega un papel relevante en la educación. En especial, en la neurociencia educativa, donde cobra una relevancia fundamental para la reforma del currículo y para el desarrollo profesional de los docentes.

\section{Aprendizaje y neurociencias: análisis de datos multimodales}

Comprender los mecanismos cerebrales necesarios para el procesamiento cognitivo y el aprendizaje, es importante para adaptar las metodologías de aprendizaje, a fin de atender temas específicos de los procesos de aprendizaje o crear intervenciones para estudiantes con necesidades específicas (Blikstein y Worsley, 2016).

Además, conocer los estados cerebrales asociados con el aprendizaje, puede contribuir a mejorar nuestra comprensión de los procesos de aprendizaje y, en consecuencia, puede mejorar el aprendizaje en si mismo. De aquí, que las neurociencias sean de tanta importancia en el estudio de los procesos de aprendizaje.

En este sentido, Ninaus et al. (2014) plantean que los resultados de las $L A$, basados en rastros neurofisiológicos, podrían ser útiles en el aprendizaje para evaluar, y optimizarlo a través del uso de un algoritmo basado en neurometrías de señales neurofisiológicas, que permita identificar si un usuario aún está aprendiendo o terminó de aprender una información dada. El algoritmo, al estar basado en un marco de análisis de señales neurofisiológicas de aprendizaje, podría identificar diferentes procesos cognitivos durante el aprendizaje.

Por otro lado, para estudiar los procesos cognitivos asociados con el aprendizaje, se requiere un estudio más específico dentro de la extensión de las neurociencias, término acuñado como neurociencia cognitiva desde finales de 1970, con el fin de explorar realmente los procesos de aprendizaje desde la perspectiva de la naturaleza humana a través de la neurociencia (Immordino-Yang y Damasion, 2008). 
La relación existente entre la ciencia del aprendizaje y el LA, formulan un papel relevante de corresponsabilidad, al también informar desde su campo de investigación cómo evoluciona LA a lo largo del tiempo. Los laboratorios, por lo general, sirven para realizar estudios que ayudan a verificar los patrones vistos en entornos de la vida real, exponiéndolos a condiciones controladas, por tal motivo la ciencia del aprendizaje puede informar el desarrollo de la analítica del aprendizaje a través de la provisión de teorías y metodologías, que ayudarán a avanzar en ambos campos. Las LA pueden ayudar a cerrar la brecha entre la neurociencia, la psicología y la educación al proporcionar una forma de observar el comportamiento de los estudiantes mientras aprenden. La combinación de LA y la ciencia del aprendizaje, por lo tanto, tiene el potencial de proporcionar formas más poderosas para monitorear y apoyar a los estudiantes a medida que aprenden (Lodge y Corrin, 2017).

En la actualidad, la analítica del aprendizaje requiere una combinación de datos provenientes de múltiples fuentes para la creación de conocimiento, esto ha provocado un mayor interés en la multimodalidad y, en consecuencia, en el análisis de datos multimodales (Di Mitri et al., 2018).

Etimológicamente, el prefijo "multi" se refiere a "más de uno", mientras que el término "modal" significa tanto "modalidad" como "modo". En otras palabras, la multimodalidad hace referencia a múltiples modalidades. Aún más, de acuerdo con Nigay y Coutaz (1993) la modalidad se refiere al tipo de canal de comunicación utilizado por dos agentes para transmitir y adquirir información, lo que define el intercambio de datos. De esta manera, el modo es el estado que determina el contexto en el que se interpreta la información.

La información contextual que caracteriza a los datos multimodales resulta muy útil para las analíticas del aprendizaje. Además, es necesario acotar que recientemente es posible recopilar y procesar estos datos en tiempo real a una escala sin precedentes, haciendo uso de sensores con los cuales es posible capturar eventos observables durante el proceso de aprendizaje, como el comportamiento del alumno y su contexto (Reimann, 2016).

Por su parte, Di Mitri et al. (2018) señalan que los recientes desarrollos tecnológicos, como la internet de las cosas (interconexión digital de objetos), los sensores portátiles, el almacenamiento de datos en la nube y el aumento del poder de cómputo para procesar y analizar grandes conjuntos de datos, han facilitado la recopilación y análisis de datos.

En el caso de los sensores, su uso en las analíticas del aprendizaje es fundamental para recopilar mediciones de alta frecuencia y micro-eventos conductuales; como por ejemplo: movimientos, habla, lenguaje corporal o respuestas fisiológicas (Di Mitri et al., 2018). 
Se hace necesario considerar que, en el proceso de aprendizaje, los eventos conductuales (medibles) coexisten con atributos latentes (no medibles), como las cogniciones o las emociones del aprendiz. Estos últimos no son observables ni cuantificables para los sensores, sino que deben ser estudiados e interpretados por los investigadores (Reimann 2016).

De acuerdo con Immordino-Yang y Damasion (2008), quienes indican, que al analizar los procesos fisiológicos, producidos por el pensamiento emocional de un alumno dentro de un salón de clase, es posible describir lo que realmente sucede en el sujeto alumno desde el punto de vista emocional, estos resultados proporcionan una nueva base para la innovación en el diseño de entornos de aprendizaje, ya sean presencial o virtual.

Precisamente, este aspecto de los datos multimodales puede ser interpretado por la neurociencia, mediante la conversión de datos biológicos, producidos por la actividad cognitiva de los alumnos durante el aprendizaje en datos digitales almacenables y organizados en conjuntos fáciles de utilizar (Reimann, 2016).

En consecuencia, al examinar todas estas modalidades de detección y evaluación aplicadas en las analíticas del aprendizaje multimodal, es posible identificar tres áreas de conocimiento relacionadas entre sí: (1) la evaluación del conocimiento de los estudiantes; (2) la evaluación de las emociones; y, (3) la medición fisiológica de los estudiantes (Blikstein y Worsley, 2016).

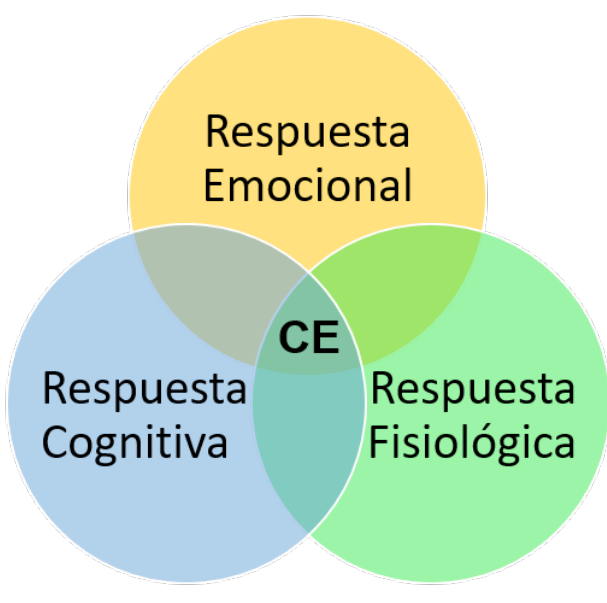

Figura 1. Áreas del conocimiento de las analíticas del aprendizaje multimodal. CE: caracterización del estudiante. Gráfica creada a partir de las interpretaciones de Blikstein y Worsley, 2016. Fuente: Elaboración propia. 
Stevens, Galloway y Berka en Blikstein y Worsley (2016) estudiaron estas relaciones con electroencefalogramas (EEG). En concreto, estudiaron las relaciones entre respuesta cognitiva, la distracción y el compromiso (respuesta emocional), descubriendo que a medida que aumentaba el nivel de habilidad de un estudiante, la carga de trabajo seguía siendo la misma.

En el centro de estas formas de evaluación se formula la caracterización de los estudiantes (CE) (ver figura 1), mientras que las relaciones subyacentes permiten generar modelos útiles a partir de grandes conjuntos de datos cuantitativos (Blikstein y Worsley, 2016).

Es necesario destacar que, en las diferentes formas de evaluación de los estudiantes, lo que varía es la fuente de los datos en bruto y cómo esos datos se traducen en datos computables. Una vez que se ha completado la traducción, los datos se pueden procesar utilizando algoritmos de aprendizaje automático (Blikstein y Worsley, 2016).

\section{Aplicaciones de datos EEG en la analítica del aprendizaje}

Hasta ahora la adopción de técnicas de EEG en educación ha sido marginal. Una de las razones, como señalaron Ansari, Coch y de Smedt (2011), es la brecha entre la investigación básica y la investigación aplicada, que complica la comunicación e intercambio de conocimiento entre educación y neurociencia cognitiva.

Sin embargo, la creciente disponibilidad de dispositivos de bajo costo, como el EEG, ha renovado el interés en la posibilidad de que la neurociencia cognitiva informe sobre la enseñanza y el aprendizaje (Durall, Leinonen, Gros y Rodriguez-Kaarto, 2017).

Antes se empleaban dispositivos EEG de grado médico, que representaban altos costos económicos y requerían de un entrenamiento médico para su uso. En la actualidad, existen en el mercado dispositivos EEG inalámbricos de bajo costo, que abren la posibilidad a toda la comunidad de investigación, para procesar y adquirir datos de la actividad cerebral con recursos accesibles (Das et al., 2014). Por ejemplo, los EEG portátiles con tecnología inalámbrica, se pueden usar para monitorear la lectura de la actividad cerebral en un aula. Su portabilidad hace más fácil la medición del sujeto; $y$, además, nos permite medir en un entorno real, en el que se procesa el aprendizaje. 
De acuerdo con Rogers (2016) en un estudio de validez, se ha demostrado que los datos derivados de un sistema inalámbrico de un solo canal de la marca NeuroSky ThinkGear, son comparables al EEG registrado en equipos convencionales de laboratorio. Adicionalmente, en su investigación se evaluó la confiabilidad de este equipo, resultando ser confiable en su sensor para la lectura de las bandas de frecuencia delta, theta, alfa y beta.

El dispositivo de hardware EEG Mindwave de la marca Neurosky está integrado por un solo sensor, que permite crear conjuntos de datos a partir de la lectura de la actividad cerebral. Este dispositivo funciona usando un hardware con un sensor para el lóbulo frontal, con el que se leen los datos de la actividad cerebral en un solo canal. Para el manejo de los datos adquiridos se emplea un software de enlace de datos, que opera por comunicación inalámbrica bluetooth, denominado Thinkgear. Para la estructuración de estos datos se emplea el software Neuroexperiementer que los almacena y los grafica (Mellender, 2015).

Una de las ventajas de esta herramienta es que permite representar en tableros los datos de las señales alfa, beta, delta y teta en sus niveles altos y bajos, además de los niveles de atención y meditación de una sesión. Los datos que se adquieren y organizan con el software el Neuroexperimenter provienen de las señales fisiológicas que se producen en el cerebro, y el hardware Neurosky las entrega al software de forma de datos EEG cuantificados, más conocidos como qEEG. Los datos se entregan al software escalados en un rango de 0 a 100 unidades, lo que permite una operatividad más eficiente. La solución del Neurosky junto con el Neuroexperimenter, permite recopilar las mediciones para el análisis e informe, la cual atiende precisamente a los atributos del LA (Mellender, 2015).

Los informes provenientes de las señales de la actividad cerebral, dentro del software Neuroexperimenter, se representan por líneas de tendencia, para poder realizar una inspección visual de las señales alfa, beta, teta y delta. Una de las ventajas de la herramienta Neurosky es que incluye algoritmos para construir las representaciones de las ondas cerebrales, que representan los niveles de atención y meditación de cada sujeto. El conjunto de datos representados en el tablero del software, son suficientes para analizar los procesos de aprendizaje de alumnos dentro de un salón de clase (Mellender, 2015). 


\section{Cerrando la brecha: LA y las neurociencias}

Se ha comprobado la importancia de las mediciones fisiológicas y cognitivas para el estudio caracterización del estudiante y de sus procesos de aprendizaje. Sin embargo, poco se menciona la importancia de estudiar las emociones y su relación con el aprendizaje.

Las emociones son procesos innatos que poseen todos los seres humanos desde su nacimiento. De acuerdo con Salas, en Linarez (2016), las emociones son uno de los elementos clave para comprender los procesos de aprendizaje de un individuo.

Todo esto acentúa la relevancia de poder leer las ondas electromagnéticas producidas por los rastros fisiológicos, como fundamento para realizar procesos de adquisición de datos de la actividad cerebral de los alumnos, mientras asisten a un curso tradicional.

Es precisamente en el campo de las neurociencias donde existe un reducido número de trabajos de investigación; por ejemplo, solo unos pocos estudios han examinado la carga cognitiva durante el aprendizaje con tecnología educativa. Esta omisión puede atribuirse hasta ahora por la falta de acceso de recursos, tales como los electroencefalogramas (EEG), debido al costo de estas tecnologías, así como a la naturaleza intrusiva de los electrodos, además del requerimiento de docenas de electrodos húmedos.

Este sustento respalda la posibilidad de incluir a las neurociencias en los procesos de LA, donde se puede buscar crear conjuntos de datos medibles para identificar y cuantificar las señales fisiológicas, producto de las emociones, para que a partir de ahí se construyan LA de fuentes externas a los sistemas empleados tradicionalmente como LMS, CMS o MOOC's.

De esta manera, las LA pueden ayudar a cerrar la brecha existente entre la neurociencia, la psicología y la educación al proporcionar una forma de observar el comportamiento de los estudiantes, mientras aprenden fuera del laboratorio. La combinación de análisis de aprendizaje y la ciencia del aprendizaje, por lo tanto, tiene el potencial de proporcionar formas más poderosas para monitorear y apoyar a los estudiantes a medida que aprenden (Lodge y Corrin, 2017).

Los desarrollos tecnológicos recientes han mejorado el EEG en tres formas clave: el costo se ha reducido; se puede usar un número menor de electrodos; y, los electrodos son secos, siendo hoy en día tan efectivos como los húmedos (Mills et al., 2017). 
Estos atributos permiten llevar equipos EEG dentro de un salón de clase real sin alteración alguna. Los avances de portabilidad, que plantean las nuevas tecnologías, permiten que los nuevos dispositivos EEG abran la posibilidad de almacenar información biológica de las lecturas de la actividad cerebral en grandes conjuntos de datos, que pueden ser analizados como estructuras para LA (Mills et al., 2017).

Sería de gran beneficio si estos datos se pudieran poner a disposición de los investigadores y su adquisición fuera planificada en coordinación con los proyectos de investigación basados en LA. En este sentido, Reimann (2016) declara que los métodos para la minería de procesos son particularmente relevantes en el contexto de las neurociencias. Esto no solo ayudaría en la realización de proyectos específicos, que estudien el aprendizaje a largo plazo, sino que también cambiaría la forma en que pensamos acerca de la naturaleza de los proyectos en la investigación del aprendizaje (Mandinach, 2012).

\section{Metodología}

Esta investigación se dividió en tres etapas: (1) selección del grupo de estudio y preparación logística del estudio; (2) instalación, pruebas de calibración y chequeo de los equipos y del software; y, (3) resultados e interpretación de los mismos.

\subsection{Selección del grupo de estudio y preparación logística}

En la primera fase se seleccionó un grupo de estudio, en este caso fue un curso tradicional de programación de base de datos, integrado por 10 alumnos de la licenciatura en sistemas computacionales. La selección de un grupo presencial se debe a lo planteado por Alberca y Borrueco (2018), quienes sustentan que los procesos de aprendizaje comienzan por la percepción de estímulos ambientales, así como la interacción con el entorno, ya que algunos de estos elementos Ilamarán la atención de los alumnos y acapararán gran parte de los recursos neuronales disponibles; como señalan, serán los estímulos los que se procesarán, integrados con otras memorias y almacenados a largo plazo para su posterior recuperación en el caso de que fuera necesario.

Cada aula es un escenario en el que interactúan una serie de variables didácticas, que hacen de la enseñanza y el aprendizaje un acto complejo. Los docentes se relacionan con los estudiantes, los objetivos, los métodos, 
las actividades, los materiales, la evaluación y el contexto; para comprender mejor el proceso de enseñanza-aprendizaje, es preciso indagar tanto lo que sucede en las clases, como en las fuerzas que operan en profundidad bajo las actuaciones visibles de los docentes (Díaz, Martínez, Roa y Sanhueza, 2010).

En este ejercicio de investigación, las neurociencias permiten, mediante sus técnicas, abrir una ventana hacia el interior de cada alumno, a fin de hacer visible a los docentes y gestores de la educación, detalles sobre los procesos que suceden a nivel fisiológico en los estudiantes, para convertir la actividad cerebral en información relevante, dando un nuevo sustento a estos procesos y una nueva concepción sobre lo que sucede en un aula tradicional.

Continuando con la idea de Alberca y Borrueco (2018) el aprendizaje es, en definitiva, un proceso individual, dependiente de la experiencia y sumamente adaptativo, por tal motivo se realizó la lectura de la actividad cerebral de cada alumno en una sola sesión de clase, con el fin de mantener lo más próximo posible todas las condiciones del entorno, como los estados de ánimo, contenidos y recursos de apoyo para la clase; donde los estados anímicos serán uno de los recursos a considerar más influyentes en los resultados de cada lectura de actividad cerebral.

Gildardo, en Falconi y otros (2017), plantea que, con el apoyo de las neurociencias, se puede llegar a entender el origen del deseo por aprender, en la medida en que se logren determinar las causas que motivan al alumno darle la significancia al aprendizaje; es por esto, que en este estudio, se pretende realizar la medición de la actividad cerebral mediante el uso de técnicas de EEG dentro de un salón de clase, donde los alumnos estarán en condiciones completamente reales sin alteración a las actividades propias de una clase presencial. Siempre, tomando como base, que todos los alumnos que participan en el estudio se encuentran completamente sanos.

Lo más importante para un educador es entender a las neurociencias como una forma de conocer de manera más amplia al cerebro; el cómo es, cómo aprende, cómo procesa, registra, conserva y evoca una información, entre otras cosas, para que a partir de este conocimiento pueda mejorar las propuestas y experiencias de aprendizaje que se dan en el aula (Gildardo en Falconi y otros, 2017).

Para lograr la adquisición de los datos, se procedió a solicitar la autorización de los alumnos para leerles su actividad cerebral, reseñar que todos accedieron voluntariamente a participar en el estudio. De la totalidad de los estudiantes del curso, se seleccionaron 6 estudiantes al azar como muestra para realizar las pruebas (3 hombres y 3 mujeres). 


\section{2 instalación, pruebas de calibración y chequeo de los equipos y del software}

La segunda etapa consistió en instalar dentro del salón de clase un equipo de cómputo con el software Neuroexperimenter, el cual permite la recepción de datos provenientes de un dispositivo Brain Computer Interface (BCl) con aspecto de diadema, modelo MindWave de la marca Neurosky. Se realizaron las pruebas de comunicación para validar que funcionaba correctamente la comunicación vía bluetooth, y que los datos eran registrados tanto por el equipo como por el software. Es importante remarcar que la diadema de lectura de las señales fisiológicas producidas de la actividad cerebral es un EEG seco, el cual se ubica en la frente por encima de las cejas, a esta zona se le conoce como ubicación FP1 del sistema internacional 10-20 de colocación de electrodos extracraneales, que representa la posición 1 del lóbulo frontal.

Alberca y Borrueco (2018) plantean que el lóbulo frontal es la región cerebral que nos distingue como humanos y se relaciona con la organización y orquestación de los distintos procesos cognitivos; e implica, entre otras funciones ejecutivas, la capacidad de planificación de la conducta, controlar la atención, inhibir conductas inapropiadas, mantener información, manipularla y comportarse en función de ésta. Por otro lado, las mismas autoras proponen, que en el lóbulo frontal se ubica el sistema de alerta, encargado de mantener un estado óptimo de vigilia, para recibir los estímulos del entorno, los cuales principalmente vendrán de las didácticas que aplique el docente durante su sesión; los estímulos propuestos por el profesor deberán generar respuestas adecuadas, De acuerdo con Henríquez (2014) las ondas gamma y beta están asociadas con la representación de la atención, la percepción y la cognición; dichas señales en su conjunto son reacciones fisiológicas en niveles de atención, este tipo de actividad cerebral se asocia a actividades humanas como escuchar, leer y hablar, que son las más comunes realizadas por un alumno dentro de un salón de clase.

El primer autor que definió a la atención fue Williams James, quien la describió como la capacidad del ser humano para orientar su estado de conciencia hacia un determinado estímulo. En la actualidad, se propone que la atención es una función neuropsicológica básica para llevar a cabo toda actividad de tipo comportamental o cognitiva, que actúa como un filtro que permite seleccionar, priorizar, procesar y supervisar la información que recibe un humano (Ramos-Galarza y otros, 2017).

De acuerdo con Ramos-Galarza y otros (2017). La atención ha sido estudiada en diversos contextos, como en el déficit de atención en el campo educativo infantil, en el daño cerebral adquirido en adultos, en estudios longitudinales de su proceso evolutivo y mecanismos cerebrales, en intervenciones de entrenamiento en niños con trastorno por déficit de atención con 
hiperactividad, en el papel de la atención sostenida en el rendimiento de deportistas, etc. Pero, el estudio de los niveles de atención en estudiantes universitarios, mediante el empleo de técnicas EEG, dentro de un salón de clase, es un contexto que todavía se encuentra en desarrollo teórico y en estado de construcción como evidencia empírica; por tal motivo, las propuestas del estudio de los procesos de atención en estudiantes universitarios aportan un grado de relevancia. Las evidencias reflejadas -encontradas por este autordenotan hasta ahora, que en este entorno se ha dado mayor importancia al estudio de la atención en la población infantil escolar.

Esta área de oportunidad de trabajar estudios de investigación con alumnos sanos en sus actividades de clase, permite crear una propuesta para cerrar las brechas entre LA y las neurociencias, ya planteado con anterioridad por Salas en Linarez (2016), las emociones son uno de los elementos clave para comprender los procesos de aprendizaje de un individuo, ya que son el reflejo de la fisiología del cerebro, y mediante el empleo de técnicas de neurociencias se pueden construir modelos de LA para un entendimiento más claro de lo que sucede en el aula tradicional.

En LA es indispensable contar con datos cuantitativos para poder proceder. Por tanto, para lograr almacenar los datos de las señales fisiológicas se emplea el software Neuroexperimenter, que almacena 11 datos relevantes, alfa1, alfa2 beta1, beta2, gamma1, gamma2, delta, teta, meditación y atención, todos leídos con el BCl Mindwave.

Los datos obtenidos se almacenan en formato CSV (Comma-Separated Values), por medio del software Neuroexperimenter. Se recopilaron los datos correspondientes a los 6 alumnos seleccionados como muestra. Los datos que se almacenan de cada alumno representan 5 minutos de su asistencia y participación en clase, los cuales conjuntan 300 datos de cada uno de los 11 tipos planteados, lo cual representan 3300 datos generados por cada alumno, representando el total de la sesión 19800 datos. Adicional a la toma de datos de las señales fisiológicas de cada alumno, se hicieron anotaciones complementarias para presentar las observaciones con los resultados de la señal fisiológica. Dichas anotaciones se sincronizaron en tiempo, junto con los 11 grupos de datos, dando un total de 12 grupos de datos que conformaron el dataset completo.

La interpretación y las analíticas de este reporte se enfoca principalmente a realizar bajo las métricas de atención y meditación proporcionadas por la misma tecnología de Neurosky, que son almacenadas y graficadas por el mismo software Neuroexperimenter, estos datos provenientes de las métricas se entregan en una base cuantificada en escala de 0 a 100 , que brinda la posibilidad de facilitar su interpretación. 


\section{Resultados}

En el estudio se generaron conjuntos de 300 datos de las señales de atención y meditación, que representaron cantidades de 0 a 100; bajo esta estructura, de manera general, se obtuvieron los niveles de atención y meditación.

Con el fin de enfocarnos en los valores de las lecturas de atención y meditación, para que realmente reflejaran impacto, se consideraron los valores superiores a 50 unidades como criterio destacado o como valor sobresaliente, descartándose los valores menores a 50 unidades, al considerarse valores débiles en su significado para la relevancia del estudio. Como resultado, se identificó que las mujeres tuvieron un balance representativo entre los niveles de atención y meditación con un valor superior a 50, como se muestra en la tabla 1:

Tabla 1. Valores promedio de forma individual y grupal entre hombre y mujeres evaluados en un curso tradicional

\begin{tabular}{ccccc}
\hline & \multicolumn{2}{c}{ Hombres } & \multicolumn{2}{c}{ Mujeres } \\
\hline Estudiante & Atención & Meditación & Atención & Meditación \\
1 & 45 & 49 & 38 & 54 \\
2 & 21 & 65 & 28 & 54 \\
3 & 36 & 23 & 65 & 53 \\
Valor promedio & 34 & 46 & 44 & 54 \\
\hline
\end{tabular}

Fuente: Elaboración propia.

Luego se realizó la construcción de series de tiempo, haciendo un seguimiento a los niveles de atención y meditación, en correlación con los eventos representativos que realizaba el profesor de forma directa o indirecta a cada alumno. Los datos mostrados en las series de tiempo representan una duración de 5 minutos de lectura de señales cerebrales, dentro del salón de clase, sin interferir entre las relaciones del profesor con sus alumnos.

En cada lectura se identifica y se marca en la serie de tiempo las actividades del profesor como se muestran en las figuras 2 y 3 , las cuales fueron organizadas con base a las observaciones y se sincronizó en tiempo por el inicio y término de cada actividad del profesor o actividad relevante del alumno, elegida en base a la acción que representase un refuerzo de conexión de interacción profesor-alumno, o bien la actividad que representase una desconexión de relación profesor-alumno. 


\section{Alumno 1}

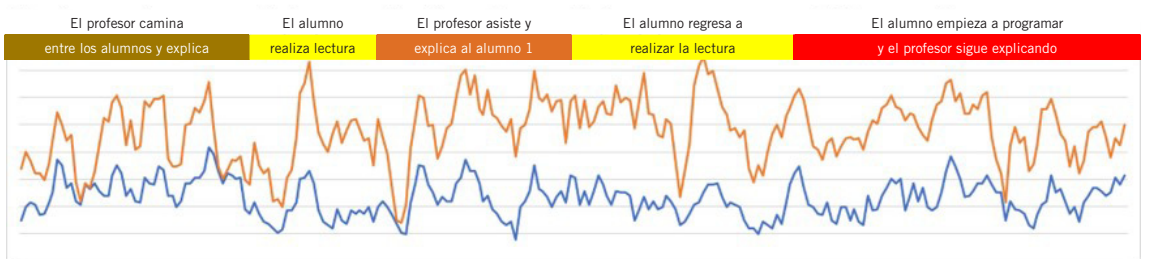

\section{Alumno 2}

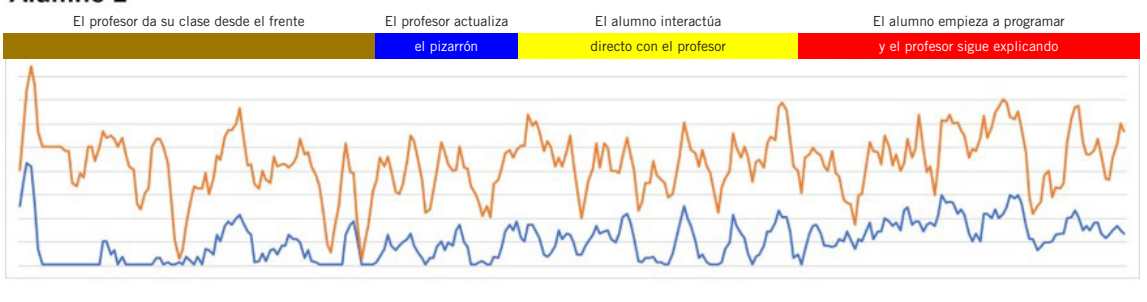

\section{Alumno 3}

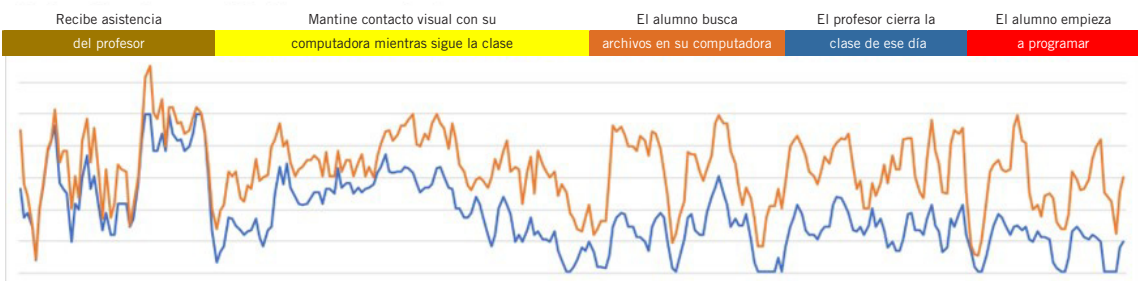

Figura 2. Lectura de señales cerebrales de la muestra de hombres durante la clase. Fuente: Elaboración propia.

Las actividades que más conllevaron a los alumnos perder intensidad de atención, fueron las lecturas de los documentos PDF, así como las actividades discursivas por parte del profesor frente al grupo. Sin embargo, cuando la actividad discursiva se realizaba entre los alumnos, era menor la pérdida de niveles de atención.

En la figura 2, se agrupa las series de tiempo de los alumnos de sexo masculino, se identifica al alumno 2, el cual presenta ausencia de atención cuando el profesor habla al frente. Mientras que cuando el profesor camina entre los alumnos, el alumno 1 demuestra nivel balanceado de atención y meditación. 


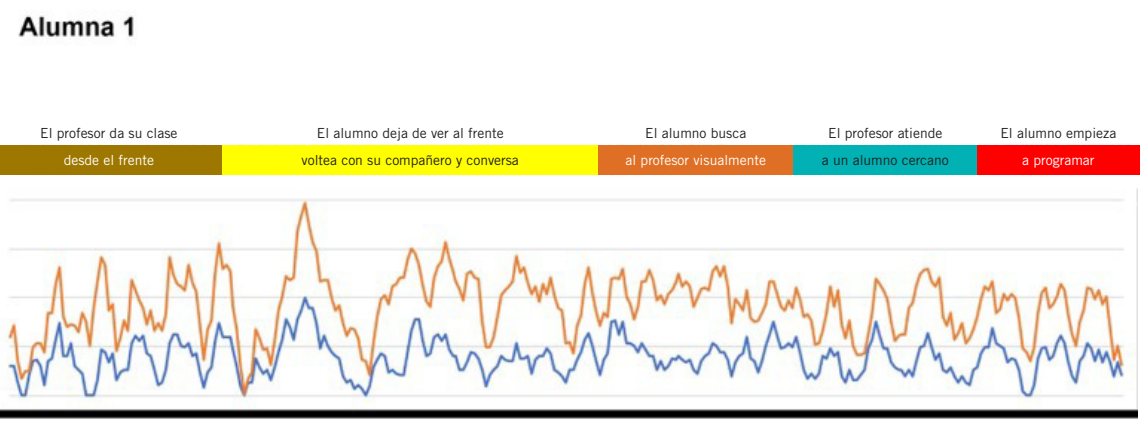

Alumna 2

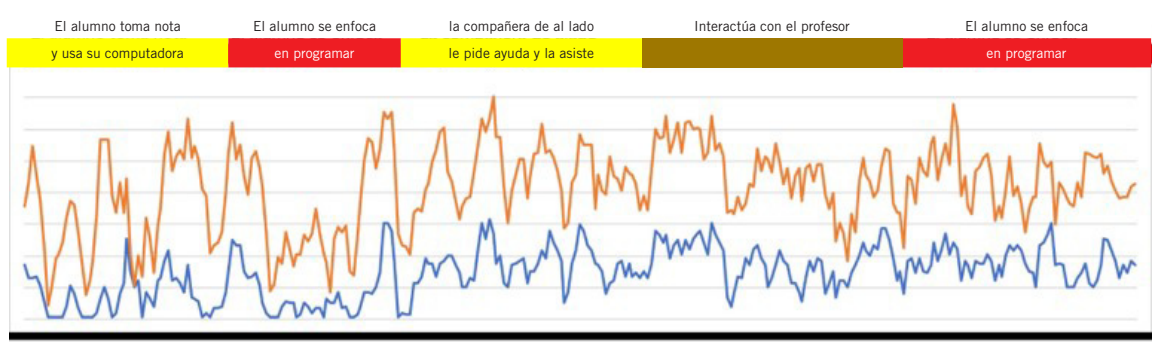

\section{Alumna 3}

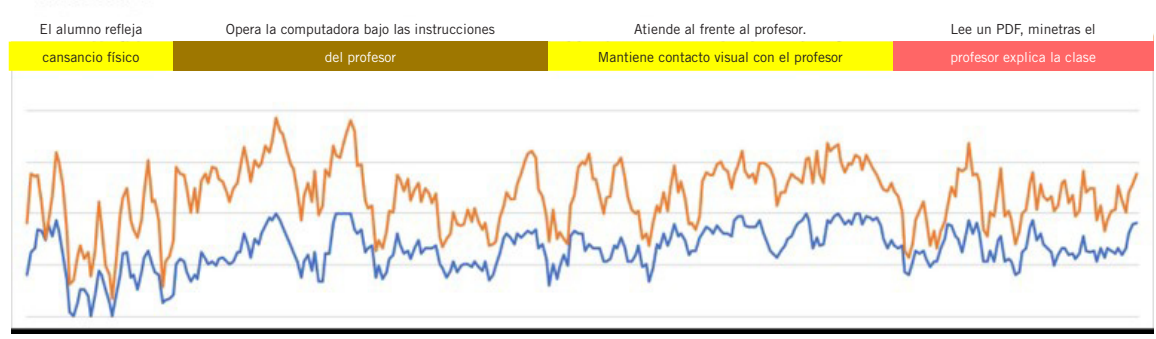

Figura 3. Lectura de señales cerebrales de la muestra de mujeres durante la clase. Fuente: Elaboración propia.

\section{Discusión}

Romero y otros (2018) declaran que la atención no constituye un proceso cerebral único, sino que existen diferentes redes atencionales donde intervienen diferentes regiones cerebrales; adicional a ello, en cada sujeto existen mecanismos inconscientes que ayudan a mantener la atención, que puede visualizarse mediante este tipo de ejercicios, ya que se puede identificar qué alumnos realmente tienen buenos hábitos de atención durante la clase 
y cuáles no. Esto puede llegar a ser un buen referente para que los docentes adecuen sus actividades dentro del salón de clase tanto en lo particular de cada alumno como en lo general de todo el grupo, ya que podremos entender a las nuevas generaciones de manera más precisa.

Los eventos identificados en ambos sexos que causaron un deterioro de la atención, se produjeron cuando el profesor tuvo una lejanía física, esto es, cuando se ubicaba al frente para llevar a cabo su práctica discursiva de los contenidos del curso, así como al comentar sobre ciertos casos específicos. Cuando el profesor atendía dudas de alumnos, los niveles de atención se vieron afectados a pesar de no ser el alumno el que hizo la pregunta. Esto puede ser debido al contacto físico que lograba el docente en una zona específica del grupo.

En general, los alumnos estuvieron realizando actividades en paralelo a lo que proponía el profesor, algunas en atención, a indicaciones del propio docente y otras ajenas a lo que solicitaba el profesor, como el caso de los alumnos 1 y 3 , tanto del sexo masculino como del sexo femenino, como se demostró durante la toma de datos. Cabe recalcar que las actividades de lecturas de documentos PDF dentro del salón de clase fueron detonantes para que los alumnos perdieran atención e iniciaran procesos de letargo, reflejando altos niveles de meditación y llevando a un decremento sostenido en los valores de atención.

De igual manera se identificó, que los alumnos que estuvieron en mejores condiciones en los procesos de aprendizaje, presentaban una cercanía en los valores de meditación y atención; en especial, cuando realizaban tareas específicas como preguntar, responder, programar, así como atender a información nueva en el pizarrón.

Ahora bien, las interrupciones de compañeros se reflejaron con cambios de ritmo en las lecturas de atención y un incremento en los niveles de meditación.

\section{Conclusiones}

La analítica del aprendizaje tiene como objetivo principal comprender y mejorar el aprendizaje y los entornos en los que se produce. Sin embargo, el aprendizaje es un proceso complejo en el cual intervienen múltiples factores; por tal motivo, se hace necesario usar herramientas, técnicas y métodos de diversos campos de investigación para estudiarla. 
Así, las analíticas del aprendizaje vienen a nutrirse de múltiples campos, haciendo uso de los conceptos y métodos que permiten obtener, describir, analizar e interpretar datos sobre los alumnos, sus procesos de aprendizaje y sus contextos.

Para las analíticas del aprendizaje el contexto es de suma importancia. En este sentido los datos que se obtienen del estudio del aprendizaje tienen un carácter multimodal caracterizado por poseer información contextual.

En la actualidad, estos datos se recopilan y procesan en tiempo real a una escala sin precedentes, haciendo uso de nuevas tecnologías como sensores y el internet de las cosas, con los que es posible extraer datos de eventos observables durante el proceso de aprendizaje (como las respuestas fisiológicas del alumno y su contexto).

Al analizar las respuestas fisiológicas en un alumno, en especial con EEG; es posible describir, desde el punto de vista emocional, lo que realmente sucede en el sujeto alumno. Estos resultados proporcionan una nueva base para la innovación en el diseño de entornos de aprendizaje, ya sean presenciales o virtuales.

En esta investigación se utilizó un dispositivo portátil EEG para el estudio de estudiantes en el aula. Con base a los resultados de estos datos, identificamos que los alumnos estuvieron en mejores condiciones para los procesos de aprendizaje, cuando se realizaron tareas específicas como preguntar, responder, programar, así como atender a información nueva en el pizarrón.

Es necesario comprender los mecanismos cerebrales usados por los estudiantes durante estas actividades para el procesamiento cognitivo y el aprendizaje, de manera que se puedan adaptar las metodologías de aprendizaje.

El avance tecnológico actual ha permitido que los costos de los equipos y recursos se estén reduciendo de manera sustancial, a medida que los métodos de LA encuentran su lugar en las escuelas y en las universidades.

Con todas estas herramientas a la mano, las LA pueden ayudar a cerrar la brecha existente entre la neurociencia, la psicología y la educación, al proporcionar una forma de observar el comportamiento de los estudiantes, mientras aprenden fuera del laboratorio. 


\section{Referencias bibliográficas}

Alberca, E. y Borrueco, M, (2018). Aprendizaje: el cerebro en el aula. Ciencia e innovación docente en el aprendizaje de lenguas extranjeras. Ediciones Egregius.

Amo, D. y Santiago, R. (2017) Learning Analytics. La narración del aprendizaje a través de los datos. Barcelona: Editorial UOC.

Ansari, D. , Coch, D. y De Smedt, B. (2011). Conexión entre la educación y la neurociencia cognitiva: ¿hacia dónde nos llevará el viaje? Filosofía y teoría educativa 43(1), 37-42. https://doi.org/10.1111/j.1469-5812.2010.00705.x

Banihashem S. K., Aliabadi, K., Pourroostae, S., Delaver A, Nili Ahmadabadi, M. (2018). Learning Analytics: A Systematic Literature Review, Interdisciplinary Journal of Virtual Learning in Medical Science. 9(2). https://doi.org/10.5812/ijvIms.63024.

Blikstein, P. y Worsley, M. (2016). Multimodal Learning Analytics and Education Data Mining: Using Computational Technologies to Measure Complex Learning Tasks. Journal of Learning Analytics, 3(2), 220-238. https://doi.org/10.18608/jla.2016.32.11

Corrin, L., Alhadad, S., Jones, H. y Colvin, C. (2018). Where is the field of learning analytics heading? Reflections from the Learning Analytics \& Knowledge Conference. Recuperado de: https://bit.ly/2V3pkKR.

Das, R., Chatterjee, D., Sinharay, A. y Sinha, A. (2014). Cognitive Load Measurement - A Methodology to Compare Low Cost Commercial EEG Devices. Advances in Computing, Communications and Informatics, ICACCI International Conference. IEEE pp.11881194.

Di Mitri, D., Schneider, J., Specht, M., y Drachsler, H. (2018). From signals to knowledge: A conceptual model for multimodal learning analytics. Journal of Computer Assisted Learning, 34(4), 338-349. https://doi.org/10.1111/jcal.12288

Díaz, C., Martínez, P., Roa, I y Sanhueza, M.G. (2010). Los docentes en la sociedad actual: sus creencias y cogniciones pedagógicas respecto al proceso didáctico, Polis. Revista Latinoamericana, 25.

Durall, E., Leinonen, T., Gros, B., y Rodriguez-Kaarto, T. (2017). Reflection in Learning through a Self-monitoring Device: Design Research on EEG Self-Monitoring during a Study Session. Designs for Learning, 9(1), 10-20. https://doi.org/10.16993/dfl.75

Falconi, A., Alajo, A., Cueva, M., Mendoza, R. Ramírez, S. y Palma, E. (2017). Las neurociencias. Una visión de su aplicación en la educación. Revista Órbita Pedagógica 4(1), 61-74

Ferguson, R. y Clow, D. (2017). Where is the evidence? A call to action for learning analytics. In: LAK '17 Proceedings of the Seventh International Learning Analytics \& Knowledge Conference, ACM International Conference Proceeding Series, pp. 56-65. New York, USA.

Henríquez, C (2014). Memoria de Trabajo de Fin de Máster Estudio de Técnicas de análisis y clasificación de señales EEG en el contexto de Sistemas BCl (Brain Computer Interface). Master Universitario en Investigación e Innovación en TIC. Universidad Autónoma de Madrid. Escuela Politécnica Superior. Departamento de Ingeniería Informática, pp 17-19.

Immordino-Yang, M. H. y Damasio, A. (2008). We Feel, Therefore We Learn: The Relevance of Affective and Social Neuroscience to Education. Mind, Brain, and Education, 1(1), 3-10. https://doi.org/10.1111/j.1751-228x.2007.00004.x 
Linarez, G. (2016). Aprendizaje significativo y neurociencia: la conexión del siglo XXI. Revista Iberoamericana de Producción Académica y Gestión Educativa, 4. 116-141

Lodge, J. M., y Corrin, L. (2017). What data and analytics can and do say about effective learning. Npj Science of Learning, 2(1). https://doi.org/10.1038/s41539-017-0006-5

Lodge, J., Hoovarth, J. y Corrin, L. (ed.) (2018) Learning Analytics in the Classroom: Translating Learning Analytics Research for Teachers. (1st Edition). London: Taylor and Francis. https://doi.org/10.4324/9781351113038

Mandinach, E.B. ( 2012 ). Un momento perfecto para el uso de datos: usar la toma de decisiones basada en datos para informar la práctica . Psicólogo de la educación , 47(2), 71 - 85

Mellender, F. (2015). NeuroExperimenter. Fred Mellender's Home Page. Recuperado de https:// bit.ly/2CuC9li

Mills, C., Fridman, I., Soussou, W., Waghray, D., Olney, A., y D'Mello, S.K. (2017). Put your thinking cap on: detecting cognitive load using EEG during learning. LAK. 80-89

Nigay, L., y Coutaz, J. (1993). A design space for multimodal systems: Concurrent processing and data fusion. Proceedings of the SIGCHI Conference on Human Factors in Computing Systems-CHI 93, (January 1993), 172-178. http://doi.org/10.1145/169059.169143

Ninaus, M., Kober, S. E., Friedrich, E. V., Neuper, C. y Wood, G. (2014). The Potential Use of Neurophysiological Signals for Learning Analytics. 2014 6th International Conference on Games and Virtual Worlds for Serious Applications (VS-GAMES). Valleta: Malta. https://doi.org/10.1109/vs-games.2014.7012169

Ramos-Galarza, C., Paredes, L., Andrade, S., Santillán, W. y González,L (2017). Sistemas de Atención Focalizada, Sostenida y Selectiva en Universitarios de Quito-Ecuador. Revista Ecuatoriana de Neurologia 25(1-3):34-38

Reimann, P. (2016). Connecting learning analytics with learning research: the role of design-based research. Learning: Research and Practice, 2:2, 130-142. https://doi.org/10.1080/ 23735082.2016.1210198

Rogers, J. M., Johnstone, S. J., Aminov, A., Donnelly, J. y Wilson, P. H. (2016). Test-retest reliability of a single-channel, wireless EEG system. International Journal of Psychophysiology, $106,87-96$

Romero, C., Luna, J.M., Bogarín, A., Luque, M., y Gonzalez. P. (2018) Análisis del nivel de atención de los alumnos en clase utilizando Neurosky's mindwave mobile. Revista de Innovación y Buenas Prácticas Docentes. 5, 57-62 Recuperado de: https://bit.ly/2Wckc3r

Siemens, G. (2013). Learning analytics: The emergences of a discipline. American Behavior Scientist. 57(10) 1380-140.

Siemens, G. (04, marzo, 2016) Neuroscience and Learning Analytics: a historic leap in understanding learning. [audio en Podcast]. Recuperado de: https://bit.ly/2IQAHiY

Society for Learning Analytics Research (SoLAR). (s.f.) Recuperado de https://bit.ly/2DDFtMc

Stewart, C. (2017). Learning Analytics: Shifting from theory to practice. Journal on Empowering Teaching Excellence, 1(1), 95-105. https://doi.org/10.15142/T3G63W

Tseng, S., Yu, L. y Wu, H. (2014). Measuring Engagement: Student Profiling and the Effects of Remedial Learning Counseling. International Workshop on Learning Analytics and Educational Data Mining (LAEDM 2016) In conjunction with CRIWG/CollabTech, 14-17.

Zadina, J. (2015) The emerging role of educational neuroscience in education reform. Psicología Educativa, 21, 71-77. Recuperado de https://bit.ly/2PC9DUY 